\title{
SMOKE DETECTION IN VIDEO FOR EARLY WARNING USING STATIC AND DYNAMIC FEATURES
}

\author{
Ashish A. Narwade ${ }^{1}$, Vrishali A. Chakkarwar ${ }^{2}$ \\ ${ }^{1}$ Research Scholar, Computer Science \& Engineering, Govt. College Of Engineering Aurangabad, Maharashtra, \\ India, aanarwade@gmail.com \\ ${ }^{2}$ Assistant Professor, Computer Science \& Engineering, Govt. College Of Engineering Aurangabad, Maharashtra, \\ India,vrush.a143@gmail.com
}

\begin{abstract}
An image processing approach for detection of smoke in video using static and dynamic features is proposed in this paper. As the conventional smoke detection such as particle sampling, smoke, temperature, Humidity Sensors needs to be close to the source of the smoke for detection, also they have weakness in wide coverage area and low response time. In order to overcome these shortcomings, this paper presents a method based on image processing techniques, capable to identify smoke from video taken from video dataset.

The proposed detection method consists of the following steps: moving region detection, smoke color detection, detecting smoke disorder, direction and then classification phase. This will provide early warnings such as fire, thus reducing economic losses and casualties. In addition, it will help to improve the rate of smoke detection, as well as reducing the false detection rate of other suspected object. The paper is structured as follows: section 1 describes introduction part and features of smoke used to differentiate it from other suspected object. Then section 2 describes the previous work including conventional and recent research on smoke detection. Next, section 3 describes the proposed smoke detection system. Finally conclusion is given.
\end{abstract}

Index Terms: smoke detection, moving region detection, smoke features.

$* * *$

\section{INTRODUCTION}

As the smoke produced by fires is visible much before the fire flames the approach proposed in this paper focuses on the detection of smoke and provide an early warning. Fire and Smoke, if not detected early enough, can cause great harm to valuable resources for human survival, destroy the balance of earth ecosystem and worst of all they frequently cost human and animal lives. Nowadays the cases of fires going on increase resulting in a large amount of losses in terms of economy, animal and human life. Mostly such cases include fire in railways, houses, factories, aircraft, buses, office, submarines, shopping malls, etc. In case of indoor fire, the primary cause of death in victims is smoke inhalation. The smoke kills by a combination of thermal damage, poisoning and pulmonary irritation caused by carbon monoxide, hydrogen cyanide and other combustion products. So the objective is that detect the smoke using static and dynamic features, as early as possible [1]. Early fire smoke detection is essential in order to provide an effective response to emergencies and damages. The aim behind this is to provide appropriate actions to mitigate the effects of the smoke and fire can be taken. These actions generally include the evacuation of occupants from danger zone to a safe place and the initiation of extinguishment activities either automatic or manual.

The conventional mechanisms such as particle sampling, smoke, temperature, sensors and humidity sensors have weaknesses in the coverage area and response time in detecting smoke [4]. The chemical properties of particles in the air are used by sensors to raise an alarm. So suppression activities became difficult and time consuming. Hence there is a need of a mechanism with faster response and can be able to monitor a wide area. To ensure a safe security there is need of tremendous research in the area of an early warning of fire and smoke detection.

In order to provide fast response, monitor a wide area and to reduce false alarms of conventional systems, an image processing based detection work is proposed in this paper. Mostly vision based systems make use of characteristic features for identifying the non-stationary regions in video. Here we are using motion and color as the initial smoke detection steps $[1,9]$. In the first step of proposed system that is moving region detection adaptive background subtraction technique is used for identifying foreground region. In an adaptive background subtraction method, background frame is updated at each frame for subtraction in order to get the foreground region [2]. This step is able to eliminate the disturbance of stationary non-smoke region. In the second step identified region of moving object will be checked for smoke color. Now in the next steps we are using the other dynamic feature of smoke to differentiate between smoke and non-smoke objects. In third step disorder feature of smoke is calculated from the ratio of smoke region perimeter and area. Then smoke direction is computed by using the max value of moving region in each frame. Finally, on the basis of all the above steps a classification will be made, that, whether there is smoke or not in a video. 


\subsection{What is Smoke?}

Smoke is a physical and chemical process. When substances burn, chemical changes will suddenly occurs, in this process particles and gases will be generated by decomposition and transformation of the substances this constitute a smoke.

\subsection{Types of smoke features}

The proposed system is based on smoke features. It can be group into two types [1]:

\section{a. Static features}

These features remain constant continuously over a period of time. In this research we are using smoke color as static feature to seprate it from other suspected object. The most of the smoke is gray that is light gray or dark gray.

\section{b. Dynamic features}

These features changes continuously over a period of time. It includes smoke disorder area, shape, direction, etc. In this research we are using disorder and direction of smoke to seprate it from other suspected moving object.

Most of the previous smoke detection system uses only motion and color information resulting in a false alarm. Hence in this proposed paper combination of motion, color and with the dynamic features of smoke is provided to get good performance of a smoke detection in video. Also dynamic features help to reduce false detection rate and increases the accuracy of smoke detection.

\section{LITERATURE SURVEY}

Some of the previous works in smoke detection with conventional and image processing approach is as follows:

\section{A. Conventional approach}

Conventional smoke detection technique use human observation, sensors, satellite, to monitor the surroundings [4]. Some of the methods (Fig -1.) are mentioned below:-

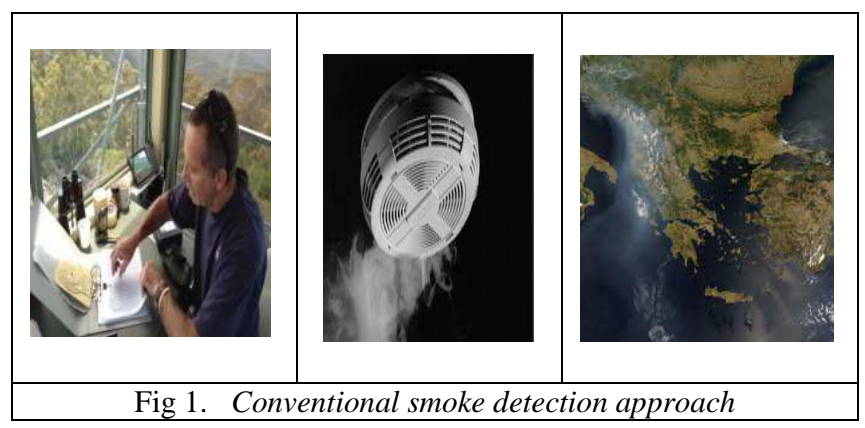

\section{a. Human observations}

The human on watch towers are made to observe the location throughout. If any fire smoke occurs he reports it. However, accurate human observation may be limited by operator fatigue, time of day, time of year, and geographic location.

\section{b. Sensors}

Sensor can cover only small area and has battery charge problem. Because they raise alarm only when smoke comes in contact it is not so much reliable as video based detection.

\section{c. Satellite and Aerial Monitoring}

Satellites based system can monitor a large area, but it has some limitations such as low image resolution, lack in real time detection, very expensive, also weather condition will seriously decrease the accurate detection as the limitations led by the long scanning period and low resolution of satellites.

\section{B. Image processing approach}

The most of smoke detection work in previous research is based on color and motion. So there are chances of false detection.

Some of the researches are mentioned below:-

\section{a. "Forest Fire Smoke Detection in Video Based on Digital Image Processing Approach"}

This work was proposed by Surapong Surit, Watchara Chatwiriya [1]. They used following steps for smoke detection: detection of area of change in the current input frame against the background image, identify regions of interest (ROIs) by connected component algorithm, calculate area of ROI by convex hull algorithm and segment the area of change from image, analysis and calculate static and dynamic characteristic and finally classifying result of above steps as smoke or non-smoke.

b. "A System for Video Surveillance and Monitoring"

This work was proposed by R. T. Collins, A. J. Lipton, and T. Kanade [2]. It uses multiple, cooperative video sensors to provide continuous coverage of people and vehicles in a cluttered environment. For estimating the moving region they have used the adaptive background estimation and subtraction method.

c. "Review of recent developments in fire detection technologies"

This work was proposed by Z. Liu and A. Kim [3]. They have used the characteristic features for smoke detection. Also they have provided some review about current techniques. They have proposed two model of fire smoke detection in video with following steps: obtaining video images, processing video images, detecting motion area, extracting fire characteristics and determining fires.

d. "Algorithm for smoke extraction in autonomous forest fire detection"

This work was proposed by Ivan Grubisic, Darko Kolaric and Karolj Skala [5]. In this work they combine motion detection, spectrum analyzing, etc. algorithm for smoke detection.

e. "Reliable smoke detection in the domains of image energy and color" 
This work was proposed by P. Piccinini, S. Calderara, and R. Cucchiara [6]. Main focus in this work is on two features: the energy variation in wavelet model and a color model of the smoke. That is Energy analysis using the discrete wavelet transform and Color analysis to detect blended smoke regions also they have used A Bayesian approach for classification.

\section{f. "Spatio-temporal analysis in smoke detection"}

This work was proposed by C. Y. Lee, C. T. Lin, and C. T. Hong [7]. It uses a Gaussian mixture model and constructs a background image for foreground detection. After this, they analyze spatial and temporal characteristics of candidate regions in video sequences, exploiting three features: edge blurring, gradual energy change and gradual chromatic configuration change. To gain proper generalization ability with respect to sparse training samples, it combines the three features using a support vector machine based classifier.

g. "Fire smoke detection in video images Using Kalman filter and Gaussian Mixture Color model”

This work was proposed by Li Ma Kaihua Wu, L. Zhu [8]. They estimated the moving object using MHI and Kalman filter. After this, they use the temporal analysis of neural network, with two features: energy variation in wavelet domain and color blending coefficient.

\section{PROPOSED SYSTEM}

The aim of this proposed work is to develop an identification system to detect an occurrence of smoke in video. In this paper, we use smoke features to conduct the smoke detection. Smoke detection process is one of subproblems in the monitoring system, whereby the object becomes the main focus is smoke. In general smoke detection process can be seen in Fig -2.

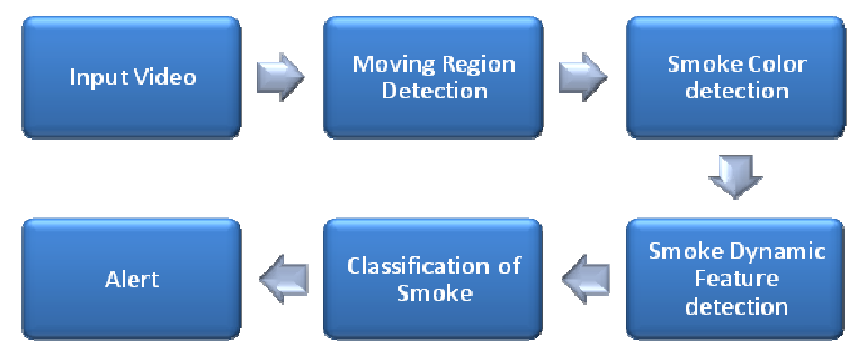

Fig -2.General block process of smoke detection.

As shown in Fig -2. Following are the main steps to do in order to get characteristics feature extraction of smoke object.

\section{step1. Moving region detection}

Smoke has a dynamic feature of motion it continuously change. So, in the first step, adaptive background subtraction method will be used to get regions of moving objects, which are candidates of possible smoke regions [2]. According to this method, a pixel is moving if:

$$
\mid I(x, y, n)-I(x, y, \text { and } n-1) \mid>T h(x, y, n)
$$

Where, $I(x, y, n)$ means intensity of pixel $(x, y)$ in $n^{\text {th }}$ frame $I$, $\mathrm{I}(\mathrm{x}, \mathrm{y}, \mathrm{n}-1)$ means intensity of pixel $(\mathrm{x}, \mathrm{y})$ in $(\mathrm{n}-1)^{\text {th }}$ frame of $\mathrm{I}$ and $\mathrm{Th}(\mathrm{x}, \mathrm{y}, \mathrm{n})$ is an adaptive threshold calculated according to:

$$
\operatorname{Th}(x, y, n+1)=\begin{array}{r}
u \operatorname{Th}(x, y, n)+(1-u)(r|I(x, y, n)-B(x, y, n)|) \\
\text { If pixel }(x, y) \text { is stationary }
\end{array}
$$

Where, $r$ is a real number greater than one, the update parameter $\mathrm{u}$ is a positive number close to one and $B(x, y, n)$ is the background image. Initial threshold $\operatorname{Th}(x, y, n)$ values are set to a pre-determined non-zero value.

An adaptive background is estimated as:

$$
B(x, y, n+1)=\begin{aligned}
& u B(x, y, n)+(1-u) I(x, y, n) \\
& B(x, y, n) \quad \text { If pixel }(x, y) \text { is stationary } \\
& \text { If pixel }(x, y) \text { is moving }
\end{aligned}
$$

Where, $\mathrm{B}(\mathrm{x}, \mathrm{y}, \mathrm{n})$ is the intensity of the estimated background related to the frame $\mathrm{n}$ at the position $(\mathrm{x}, \mathrm{y})$. The update parameter $\mathrm{u}$ is a positive real number close to one. Initially, $\mathrm{B}(\mathrm{x}, \mathrm{y}, 0)$ is set to the first image frame $\mathrm{I}(\mathrm{x}, \mathrm{y}, 0)$. Finally an estimated background image is subtracted from the current image to detect moving regions as:

$$
|I(x, y, n)-B(x, y, n)|>T h(x, y, n)
$$

In this way we will get the non-stationary regions that are the possible candidates of smoke to process in further processing. In The next steps with moving region in hand we check it for smoke using feature of smoke, to seprate out non-smoke area.

\section{step2. Smoke color detection}

In this step moving region computed in previous step is used for checking against smoke color. The smoke usually shows grayish color in process of burning material. For this the average color value of RGB in each pixel is estimated with following equation [1]:

$$
A v g x y=(R x y+G x y+B x y) / 3
$$

Next, absolute difference of RGB is compare with Th to find gray smoke color with following equation:

$$
\begin{gathered}
\text { If } \mid R x y-\text { avgxy } \& \mid \text { Gxy-avgxy } \& \mid B x y-\text { avgxy } \mid<T h \\
\text { Color of smoke }
\end{gathered}
$$


Else

$$
\text { Not smoke color }
$$

step3. Dynamic features of smoke

In the proposed system dynamic characteristics of smoke such as disorder, direction is used for more accurate detection of smoke $[10,11]$. Since in the previous steps we separated the smoke color object but there might be things that confused with the smoke such as people, car, and shadows moving on, etc.

\section{a. Smoke disorder detection}

Smoke disorder means change in scale when smoke spread. When the smoke comes it spread in different directions. It is the dynamic and unique nature of smoke and all smoke show this type of nature. The Smoke disorder is calculated with following equation:

$$
\text { Disorder }=\frac{\text { Perimeter }}{\text { Area }} \geq \text { Threshold }
$$

Where, the perimeter is the sums of circumferences of smoke regions, area is the sum of smoke pixel and Threshold value is used to distinguish the other similar objects. Likewise disorder value is calculated for each frame.

\section{b. Smoke direction detection}

As smoke continuously moves in upward direction, based on this distinguishing feature of smoke we employ the following logic. In this step, if smoke direction is up then max value of binary image for each frame is used. Initially, max value for suspected region of first frame is taken. Likewise, max values for suspected region of next frames are taken. After this, we use condition that if suspected region value observed in the next frame is larger than previous frame then that is a smoke with up direction. It is represented as:

$$
v s f 1<v s f 2 \text { continue, }
$$

This process will continue until one of the next two condition holds first value equal to second value or second value less than first. It is represented as:

$$
\begin{array}{rr}
V s f 1>v s f 2 \quad \text { stop } \\
\text { Or } & \\
v s f 1=v s f 2 \quad \text { stop. }
\end{array}
$$

Where, $v s f 1$ is the max value of first frame, $v s f 2$ is the max value of next frame. It will help in differentiating smoke from other similar object. step4. Decision/Classification of smoke

In this step decision will be made on the basis of smoke features to classify the extracted region as smoke or nonsmoke. Support vector machine is a set of related supervised learning methods that analyze data and recognize features, thus it will be employed in our method for features based classification. Also the alert will be given when smoke detected for taking next action.

\section{CONCLUSIONS}

In this paper, a smoke detection in video approach combining motion, color and dynamic features of smoke is proposed. It is an efficient and responsive approach for detecting smoke in the video for early detection of fire smoke. This approach can be used for detecting smoke in indoor and outdoor area. In our method we are taking into the consideration the static and dynamic nature of smoke for effective and accurate detection.

\section{ACKNOWLEDGEMENT}

Authors feel huge gratitude to all the faculty members of the Computer Science Department of Government College of Engineering, Aurangabad, India, for their enthusiasm and help for the preparation of this paper. They thank Dr. P. S. Advani, Principal, Government College of Engineering, Aurangabad, for his constant support to our work.

\section{REFERENCES}

[1] Surapong Surit, Watchara Chatwiriya , "Forest Fire Smoke Detection in Video Based on Digital Image Processing Approach with Static and Dynamic Characteristic Analysis", in IEEE First ACIS/JNU International Conference on Computers, Networks, Systems and Industrial Engineering, pp.35-39, 2011,

[2] R. T. Collins, A. J. Lipton, T. Kanade, "A System for Video Surveillance and Monitoring", Proc. of American Nuclear Society 8th Int. Topical Meeting on Robotics and Remote Systems, Pittsburgh, PA, Apr.25-29, 1999.

[3] Z. Liu and A. Kim, "Review of recent developments in fire detection technologies", in Journal of Fire Protection Engineering, vol. 13, no. 2, pp. 129-149, May 2003.

[4] Vipin V, "Image Processing Base Forest Fire Detection", in IJETAE, Volume 2, Issue 2, pp. 87-94, Feb2012.

[5] Ivan Grubisic, Darko Kolaric and Karolj Skala, "Intelligent algorithm for smoke extraction in autonomous forest fire detection", Optoelectronics and Visualization Laboratory Centre for Informatics and Computing, Ruder Boskovic Institute Croatia. 
[6] P. Piccinini, S. Calderara and R. Cucchiara, "Reliable smoke detection in the domains of image energy and color", in IEEE International Conference on Image Processing (ICIP), pp. 1376-1379, October 2008.

[7] C.-Y. Lee, C.-T. Lin and C.-T. Hong, "Spatio-temporal analysis in smoke detection", in IEEE International Conference on Signal and Image Processing Applications (ICSIPA), pp. 80-83, November 2009.

[8] H. Maruta, A. Nakamura and F. Kurokawa, "A new approach for smoke detection with texture analysis and support vector machine", in IEEE International Symposium on Industrial Electronics (ISIE), pp. 15501555, July 2010.

[9] T. Truong and J. Kim. "An Early Smoke Detection System based on Motion Estimation", in International Forum on Strategic Technology, pp. 437-440, 2010.

[10] Ali Rafiee, Reza Tavakoli, Reza Dianat, Sara Abbaspour, "Fire and Smoke Detection using Wavelet Analysis and Disorder Characteristics", in IEEE, pp. 262-265, 2011.

[11] Changwoo Ha, Gwanggil Jeon and Jechang Jeong, "Vision-Based Smoke detection Algorithm for Early Fire Recognition in Digital Recording System", in Congress on Image and Signal Processing (CISP), vol. 3, pp. 95-99, May 2008.

[12] "Wildfire Smoke Detection", http://www.dti.unimi.it/genovese/wild/wildfire.html.

[13] "Wildfire Observers and Smoke Recognition Homepage", http://wildfire.fesb.hr/.

[14] "Sample fire and smoke video clips", Signal and Image Processing Group, Bilkent University, http://signal.ee.bilkent.edu.tr/VisiFire/.

\section{BIOGRAPHIES}

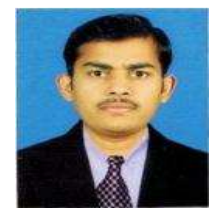

Ashish A. Narwade received Bachelor Engineering degree in Computer Science. He is currently pursuing Master Engineering degree in Computer Science from GECA, Aurangabad.

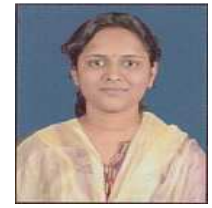

Prof. Vrishali A. Chakkarwar received Bachelor Engineering And Master Engineering in Computer Science. She is currently assistant professor in GECA, Aurangabad. Her research area includes computer Vision, Data Mining and Image Processing. She has published more than 15 international research papers. 